\title{
ROMANIAN
}

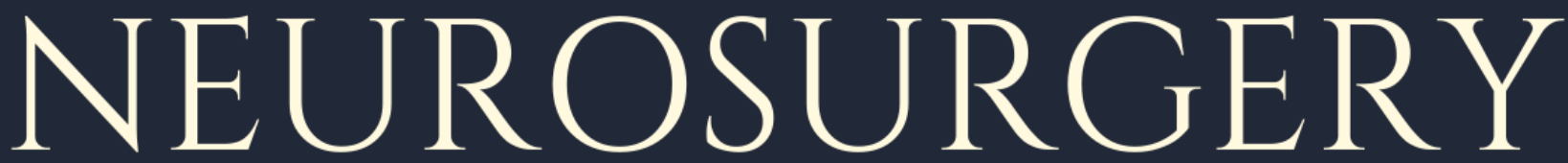

Vol. XXXIV | No. 2 June 2020

Outcome analysis of upper and lower limb motor functions after anterior cervical discectomy and fusion for degenerative cervical disc disease

\author{
Ninad N. Srikhande, V.A. Kiran \\ Kumar, N.A. Sai Kiran, Amrita \\ Ghosh, Ranabir Pal, Luis Rafael \\ Moscote-Salazar, V. Anil Kumar, \\ Vishnu Vardhan Reddy, Amit \\ Agrawal
}




\section{Outcome analysis of upper and lower limb motor functions after anterior cervical discectomy and fusion for degenerative cervical disc disease}

\author{
Ninad N. Srikhande ${ }^{1}$, V.A. Kiran Kumar ${ }^{1}$, N.A. Sai \\ Kiran ${ }^{1}$, Amrita Ghosh ${ }^{2}$, Ranabir Pal ${ }^{3}$, Luis Rafael \\ Moscote-Salazar ${ }^{4}$, V. Anil Kumar ${ }^{5}$, Vishnu \\ Vardhan Reddy ${ }^{1}$, Amit Agrawal ${ }^{1}$
}

1 Department of Neurosurgery, Narayana Medical College Hospital, Chinthareddypalem, Nellore, Andhra Pradesh, INDIA

2 Department of Biochemistry, Calcutta Medical College, 88, College street, Kolkata-700073, INDIA

${ }^{3}$ Department of Community Medicine, MGM Medical College and LSK Hospital, Kishanganj -855107, Bihar, INDIA

4 Neurosurgery-Critical Care, Red Latino, Organización

Latinoamericana de Trauma y cuidado, Neurointensivo, Bogota, COLOMBIA

5 Department of Anaesthesia, Narayana Medical College Hospital, Chinthareddypalem, Nellore, Andhra Pradesh, INDIA

\footnotetext{
ABSTRACT

Background: Anterior cervical discectomy and fusion (ACDF) is the most commonly performed surgical procedure for symptomatic cervical disc disease. In this study, we analysed the upper and lower limb motor functions after ACDF for disc prolapse in patients with degenerative cervical disc disease.

Methods: One hundred consecutive adult patients who underwent ACDF for single or two-level cervical disc prolapse during the study period (October 2015 to October 2017) were included in the study.

Results: Preoperative motor deficits in limbs were noted in $73 \%(73 / 100)$ of the patients. Enhance recovery of motor deficits was noted in $72.6 \%(53 / 73)$ of these patients and persisting motor deficits in the remaining patients (20/73-27.4\%). Five patients (5/27- 18.5\%) without any preoperative motor deficits developed motor deficits after ACDF. Detailed pre and postoperative (at the time of discharge) motor power (graded by MRC grade) in all 4 limbs (Shoulder abduction / adduction / flexion / extension, elbow flexion / extension, wrist flexion / extension, hip abduction / adduction / flexion / extension, knee flexion/extension, ankle flexion/extension) was recorded. Statistically significant improvement in motor power (as recorded at the time of discharge) was noted in all the tested muscle groups after ACDF.

Conclusion: Early improvement in preoperative motor deficits can be expected in the majority of the patients with cervical PIVD following ACDF.
}

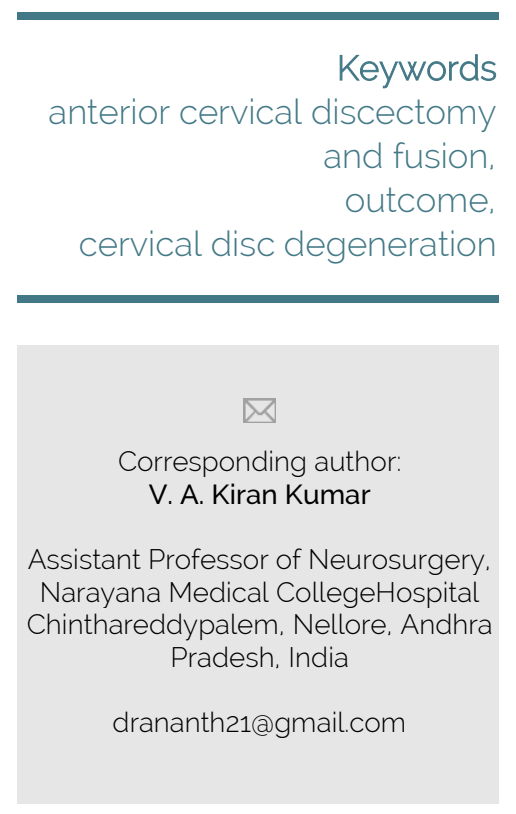

Copyright and usage. This is an Open Access article distributed under the terms of the Creative Commons Attribution Non-Commercial No Derivatives License (https://creativecommons org/licenses/by-nc-nd/4.0/) which permits noncommercial re-use, distribution, and reproduction in any medium, provided the original work is unaltered and is properly cited.

The written permission of the Romanian Society of Neurosurgery must be obtained for commercial re-use or in order to create a derivative work.

ISSN online 2344-4959 (C) Romanian Society of Neurosurgery

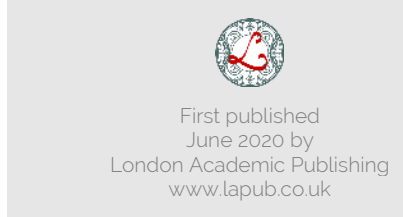




\section{INTRODUCTION}

ACDF is most commonly done to treat a symptomatic cervical PIVD. $(1,16,20,22,23,25)$ ACDF is a safe procedure and is rarely associated with postoperative complications. $(2,7,9,12,17)$ Significant proportion of patients shows remarkable recovery in motor deficits following ACDF. $(11,16)$ Authors in this publication analysed in detail the early recovery of motor deficits following ACDF for single or two level degenerative cervical PIVD.

\section{MATERIAL AND METHODS}

One hundred consecutive adult patients who underwent ACDF for degenerative cervical PIVD during the study period (October 2015 to October 2017) were included in the study. Patients with traumatic PIVD were excluded. Approval from the institutional ethical committee was taken for this study. Detailed pre and postoperative (At the time of discharge) motor power (graded by MRC grade except hand grip which was subjectively graded from 0-100\%) in all 4 limbs (Shoulder abduction / adduction / flexion / extension, elbow flexion / extension, wrist flexion / extension, hip abduction / adduction / flexion / extension, knee flexion / extension, ankle flexion / extension) were analysed.

\section{Statistical analysis}

Data analysis was done by using statistical software SPSS Statistics Version 24.0. Descriptive statistics including mean and standard deviation for continuous variables, and frequency and percentage for categorical variables were used for data expression. Appropriate tests like Chi-square test, Wilcoxon signed rank test etc. were used for checking statistically significant correlation. A probability $(P)$ value of $<0.05$ was considered significant.

\section{RESULTS}

Preoperative motor deficits in limbs were noted in $73 \%$ (73/100) of the patients. At the time of discharge, enhance recovery of motor deficits was noted in $72.6 \%(53 / 73)$ of these patients and persisting motor deficits in the remaining patients (20/73-27.4\%). Five patients (5/27- 18.5\%) without any preoperative motor deficits developed motor deficits after ACDF. Pre and postoperative (At the time of discharge) motor power in all 4 limbs is compared in Tables 1-4. Statistically significant improvement in motor power at the time of discharge was recorded in all the tested muscle groups after ACDF.

\begin{tabular}{|c|c|c|c|c|c|c|c|c|c|}
\hline \multirow{2}{*}{$\begin{array}{l}\text { Right } \\
\text { upper } \\
\text { limb }\end{array}$} & \multicolumn{4}{|c|}{ Preoperative $(n=100)$} & \multicolumn{4}{|c|}{ Postoperative $(n=100)$} & \multirow{2}{*}{ P-value } \\
\hline & Mean" & Median\# & Range $^{\#}$ & IQR & Mean" & Median" & Range $^{\#}$ & IQR & \\
\hline \multicolumn{10}{|c|}{ Shoulder } \\
\hline $\begin{array}{l}\text { Should } \\
\text { er } \\
\text { abducti } \\
\text { on }\end{array}$ & $3.80 \pm 1.4$ & 4 & $0-5$ & 2 & $4.15 \pm 1.29$ & 5 & $0-5$ & 1 & $0.001(S)^{*}$ \\
\hline $\begin{array}{l}\text { Should } \\
\text { er } \\
\text { adducti } \\
\text { on }\end{array}$ & $3.80 \pm 1.4$ & 4 & $0-5$ & 2 & $4.16 \pm 1.29$ & 5 & $0-5$ & 1 & $0.001(\mathrm{~S})^{*}$ \\
\hline $\begin{array}{l}\text { Should } \\
\text { er } \\
\text { flexion }\end{array}$ & $3.81 \pm 1.4$ & 4 & $0-5$ & 2 & $4.18 \pm 1.29$ & 5 & $0-5$ & 1 & $0.001(S)^{*}$ \\
\hline $\begin{array}{l}\text { Should } \\
\text { er } \\
\text { extensi } \\
\text { on }\end{array}$ & $3.80 \pm 1.4$ & 4 & $0-5$ & 2 & $4.17 \pm 1.32$ & 5 & $0-5$ & 1 & $0.001(S)^{*}$ \\
\hline
\end{tabular}




\begin{tabular}{|c|c|c|c|c|c|c|c|c|c|}
\hline \multicolumn{10}{|l|}{ Elbow } \\
\hline $\begin{array}{l}\text { Elbow } \\
\text { flexion }\end{array}$ & $3.73 \pm 1.5$ & 4 & $0-5$ & 1 & $4.17 \pm 1.32$ & 5 & $0-5$ & 1 & $0.001(S)^{*}$ \\
\hline $\begin{array}{l}\text { Elbow } \\
\text { extensi } \\
\text { on }\end{array}$ & $3.71 \pm 1.5$ & 4 & $0-5$ & 2 & $4.16 \pm 1.33$ & 5 & $0-5$ & 1 & $0.001(S)^{*}$ \\
\hline \multicolumn{10}{|l|}{ Wrist } \\
\hline $\begin{array}{l}\text { Wrist } \\
\text { flexion }\end{array}$ & $3.71 \pm 1.6$ & 4 & $0-5$ & 1 & $4.14 \pm 1.27$ & 5 & $0-5$ & 1 & $0.001(S)^{*}$ \\
\hline $\begin{array}{l}\text { Wrist } \\
\text { extensi } \\
\text { on }\end{array}$ & $3.72 \pm 1.6$ & 4 & $0-5$ & 2 & $4.14 \pm 1.29$ & 5 & $0-5$ & 1 & $0.001(S)^{*}$ \\
\hline $\begin{array}{l}\text { Right } \\
\text { hand } \\
\text { grip }\end{array}$ & \multicolumn{4}{|c|}{ Preoperative (Number of patients) } & \multicolumn{4}{|c|}{ Postoperative (Number of patients) } & P-value \\
\hline $0-25 \%$ & \multicolumn{4}{|l|}{11} & \multicolumn{4}{|l|}{4} & \multirow{4}{*}{$0.001(S)^{\dagger}$} \\
\hline $\begin{array}{l}25-50 \\
\%\end{array}$ & \multicolumn{4}{|l|}{11} & \multicolumn{4}{|l|}{13} & \\
\hline $\begin{array}{l}50-75 \\
\%\end{array}$ & \multicolumn{4}{|l|}{29} & \multicolumn{4}{|l|}{22} & \\
\hline $\begin{array}{l}75- \\
100 \%\end{array}$ & \multicolumn{4}{|l|}{49} & \multicolumn{4}{|l|}{61} & \\
\hline
\end{tabular}

Table 1. Comparison of preoperative and postoperative motor power in right upper limb. \#Power graded according to MRC grade.

*Obtained Using Wilcoxon signed rank test; † Obtained using Chi square test; S: Significant.

\begin{tabular}{|c|c|c|c|c|c|c|c|c|c|}
\hline \multirow{2}{*}{$\begin{array}{l}\text { Left } \\
\text { upper } \\
\text { limb }\end{array}$} & \multicolumn{4}{|c|}{ Preoperative $(n=100)$} & \multicolumn{4}{|c|}{ Postoperative $(n=100)$} & \multirow{2}{*}{$\begin{array}{l}\text { P- } \\
\text { value* }\end{array}$} \\
\hline & Mean" & Median\# & Range $^{\#}$ & IQR & Mean\# & Median" & $\begin{array}{l}\text { Range } \\
\#\end{array}$ & IQR & \\
\hline \multicolumn{10}{|l|}{ Shoulder } \\
\hline $\begin{array}{l}\text { Shoulder } \\
\text { abduction }\end{array}$ & $\begin{array}{l}3.75 \pm 1.4 \\
1\end{array}$ & 4 & $0-5$ & 2 & $4.12 \pm 1.36$ & 5 & $0-5$ & 1 & $\begin{array}{l}0.001 \\
(S)^{*}\end{array}$ \\
\hline $\begin{array}{l}\text { Shoulder } \\
\text { adduction }\end{array}$ & $\begin{array}{l}3.74 \pm 1.4 \\
1\end{array}$ & 4 & $0-5$ & 2 & $4.14 \pm 1.34$ & 5 & $0-5$ & 1 & $\begin{array}{l}0.001 \\
(S)^{*}\end{array}$ \\
\hline $\begin{array}{l}\text { Shoulder } \\
\text { flexion }\end{array}$ & $\begin{array}{l}3.72 \pm 1.4 \\
2\end{array}$ & 4 & $0-5$ & 2 & $4.13 \pm 1.34$ & 5 & $0-5$ & 1 & $\begin{array}{l}0.001 \\
(S)^{*}\end{array}$ \\
\hline $\begin{array}{l}\text { Shoulder } \\
\text { extension }\end{array}$ & $\begin{array}{l}3.72 \pm 1.4 \\
5\end{array}$ & 4 & $0-5$ & 2 & $4.13 \pm 1.32$ & 5 & $0-5$ & 1 & $\begin{array}{l}0.001 \\
(S)^{*}\end{array}$ \\
\hline Elbow & & & & & & & & & \\
\hline
\end{tabular}




\begin{tabular}{|c|c|c|c|c|c|c|c|c|c|}
\hline $\begin{array}{l}\text { Elbow } \\
\text { flexion }\end{array}$ & $\begin{array}{l}3.70 \pm 1.4 \\
8\end{array}$ & 4 & $0-5$ & 2 & $4.13 \pm 1.30$ & 5 & $0-5$ & 1 & $\begin{array}{l}0.001 \\
(S)^{*}\end{array}$ \\
\hline $\begin{array}{l}\text { Elbow } \\
\text { extension }\end{array}$ & $\begin{array}{l}3.70 \pm 1.4 \\
8\end{array}$ & 4 & $0-5$ & 2 & $4.13 \pm 1.29$ & 5 & $0-5$ & 1 & $\begin{array}{l}0.001 \\
(S)^{*}\end{array}$ \\
\hline \multicolumn{10}{|l|}{ Wrist } \\
\hline $\begin{array}{l}\text { Wrist } \\
\text { flexion }\end{array}$ & $\begin{array}{l}3.64 \pm 1.5 \\
1\end{array}$ & 4 & $0-5$ & 1 & $4.2 \pm 1.26$ & 5 & $0-5$ & 1 & $\begin{array}{l}0.001 \\
(S)^{*}\end{array}$ \\
\hline $\begin{array}{l}\text { Wrist } \\
\text { extension }\end{array}$ & $\begin{array}{l}3.64 \pm 1.5 \\
3\end{array}$ & 4 & $0-5$ & 2 & $4.14 \pm 1.3$ & 5 & $0-5$ & 1 & $\begin{array}{l}0.001 \\
(S)^{*}\end{array}$ \\
\hline $\begin{array}{l}\text { Left } \\
\text { hand } \\
\text { grip }\end{array}$ & \multicolumn{4}{|c|}{ Preoperative (Number of patients) } & \multicolumn{4}{|c|}{ Postoperative (Number of patients) } & P-value \\
\hline $0-25 \%$ & \multicolumn{4}{|l|}{12} & \multicolumn{4}{|l|}{3} & \multirow{4}{*}{$\begin{array}{l}0.001 \\
(S)^{\dagger}\end{array}$} \\
\hline $25-50 \%$ & \multicolumn{4}{|l|}{11} & \multicolumn{4}{|l|}{13} & \\
\hline $50-75 \%$ & \multicolumn{4}{|l|}{34} & \multicolumn{4}{|l|}{16} & \\
\hline $75-100 \%$ & \multicolumn{4}{|l|}{43} & \multicolumn{4}{|l|}{68} & \\
\hline
\end{tabular}

Table 2. Comparison of preoperative and postoperative motor power in left upper limb.

\#Power graded according to MRC grade

*Obtained Using Wilcoxon signed rank test; † Obtained using Chi square test; S: Significant

\begin{tabular}{|c|c|c|c|c|c|c|c|c|c|}
\hline \multirow{2}{*}{ Right lower limb } & \multicolumn{4}{|c|}{ Preoperative $(n=100)$} & \multicolumn{4}{|c|}{ Postoperative $(n=100)$} & \multirow{2}{*}{ P-value* } \\
\hline & Mean" & Median" & Range ${ }^{\#}$ & IQR & Mean" & Median" & Range ${ }^{\#}$ & IQR & \\
\hline \multicolumn{10}{|l|}{ Hip } \\
\hline Hip abduction & $3.42 \pm 1.85$ & 4 & $0-5$ & 2 & $3.90 \pm 1.59$ & 5 & $0-5$ & 2 & $0.001(S)$ \\
\hline Hip adduction & $3.43 \pm 1.88$ & 4 & $0-5$ & 2 & $3.91 \pm 1.59$ & 5 & $0-5$ & 1 & $0.001(S)$ \\
\hline Hip flexion & $3.43 \pm 1.88$ & 4 & $0-5$ & 2 & $3.91 \pm 1.59$ & 5 & $0-5$ & 1 & $0.001(S)$ \\
\hline Hip extension & $3.42 \pm 1.88$ & 4 & $0-5$ & 2 & $3.88 \pm 1.59$ & 5 & $0-5$ & 2 & $0.001(S)$ \\
\hline Knee & & & & & & & & & \\
\hline
\end{tabular}




\begin{tabular}{|l|l|l|l|l|l|l|l|l|l|}
\hline Knee flexion & $3.40 \pm 1.86$ & 4 & $0-5$ & 2 & $3.90 \pm 1.60$ & 5 & $0-5$ & 2 & $\mathbf{0 . 0 0 1 ( S )}$ \\
\hline Knee extension & $3.38 \pm 1.86$ & 4 & $0-5$ & 2 & $3.90 \pm 1.59$ & 5 & $0-5$ & 2 & $\mathbf{0 . 0 0 1 ( \mathbf { S } )}$ \\
\hline Ankle & & & & & & & & & \\
\hline Ankle flexion & $3.33 \pm 1.85$ & 4 & $0-5$ & 2 & $3.84 \pm 1.58$ & 4.5 & $0-5$ & 2 & $\mathbf{0 . 0 0 1 ( \mathbf { S } )}$ \\
\hline Ankle extension & $3.35 \pm 1.86$ & 4 & $0-5$ & 2 & $3.85 \pm 1.58$ & 5 & $0-5$ & 2 & $\mathbf{0 . 0 0 1 ( S )}$ \\
\hline
\end{tabular}

Table 3. Comparison of preoperative and postoperative motor power in right lower limb. \#Power graded according to MRC grade

*Obtained Using Wilcoxon signed rank test; S: Significant

\begin{tabular}{|c|c|c|c|c|c|c|c|c|c|}
\hline \multirow{2}{*}{ Left lower limb } & \multicolumn{4}{|c|}{ Preoperative $(n=100)$} & \multicolumn{4}{|c|}{ Postoperative $(n=100)$} & \multirow{2}{*}{ P-value* } \\
\hline & Mean & Median & Range & IQR & Mean & Median & Range & IQR & \\
\hline \multicolumn{10}{|l|}{ Hip } \\
\hline Hip abduction & $3.32 \pm 1.86$ & 4 & $0-5$ & 2 & $3.98 \pm 1.53$ & 5 & $0-5$ & 1 & $0.001(S)$ \\
\hline Hip adduction & $3.33 \pm 1.87$ & 4 & $0-5$ & 2 & $3.99 \pm 1.52$ & 5 & $0-5$ & 1 & $0.001(S)$ \\
\hline Hip flexion & $3.32 \pm 1.86$ & 4 & $0-5$ & 2 & $4.00 \pm 1.49$ & 5 & $0-5$ & 1 & $0.001(\mathrm{~S})$ \\
\hline Hip extension & $3.29 \pm 1.84$ & 4 & $0-5$ & 2 & $3.98 \pm 1.49$ & 5 & $0-5$ & 1 & $0.001(S)$ \\
\hline \multicolumn{10}{|l|}{ Knee } \\
\hline Knee flexion & $3.27 \pm 1.83$ & 4 & $0-5$ & 2 & $3.95 \pm 1.52$ & 5 & $0-5$ & 1 & $0.001(S)$ \\
\hline Knee extension & $3.27 \pm 1.84$ & 4 & $0-5$ & 2 & $3.94 \pm 1.51$ & 5 & $0-5$ & 1 & $0.001(S)$ \\
\hline \multicolumn{10}{|l|}{ Ankle } \\
\hline Ankle flexion & $3.22 \pm 1.89$ & 4 & $0-5$ & 3 & $3.88 \pm 1.55$ & 5 & $0-5$ & 2 & $0.001(\mathrm{~S})$ \\
\hline Ankle extension & $3.20 \pm 1.89$ & 4 & $0-5$ & 3 & $3.89 \pm 1.56$ & 5 & $0-5$ & 2 & $0.001(\mathrm{~S})$ \\
\hline
\end{tabular}

Table 4. Comparison of preoperative and postoperative motor power in right lower limb. \#Power graded according to MRC grade

*Obtained Using Wilcoxon signed rank test; S: Significant

\section{DISCUSSION}

Cervical PIVD is a common degenerative disc disease affecting millions of people. (24) Cervical disc herniation can occur as a result of ageing, wear and tear, or sudden stress from an accident.(5) Majority of these patients present with neck pain radiating to upper limbs. (24) Other presenting symptoms include motor deficits, stiffness in limbs, sensory deficits, paresthesias in limbs etc. $(16,24)$ Majority of the patients presenting with only neck pain or radicular pain can be managed with medicationas and conservative measures like physiotherapy, cervical collar etc. Patients with significant pain not responding to conservative measures and patients with neurological deficits like sensory/motor deficits and bladder symptoms respond well to surgery. (10, 16, 21)

ACDF is a common surgical procedure performed for symptomatic degenerative cervical disc disease. $(6,16)$ It helps to relieve the pressure on nerve roots and/or on the spinal cord, (14) thus resulting in improvement in various clinical symptoms including 
neck pain, radicular pain, motor weakness, sensory symptoms, tightness in limbs and bladder disturbances. $(10,13,16)$ Various complications reported with ACDF include dysphagia, hoarseness of voice, wound hematoma, graft migration, pseudoarthrosis, wound infection etc. (13) Patients can rarely have sensory or motor deficits after ACDF due to small risk of damage to the spinal cord, nerve roots or both. $(10,13,16)$

Improvement in neurological deficits ranging from $36-93 \%$ has been reported in various series after ACDF.(3, 13, 15, 16, 18) Lehman et al (16) reported preoperative motor deficits in $55 \%$ of the patients and reported recovery of these deficits in $95 \%$ of them at 1 year. Chiles et al(4) reported strength improvement rates ranging form $79.1 \%$ to $90.9 \%$ in various individual muscle groups of upper and lower limbs following ACDF. In the present study very high proportion of patients (73\%) presented with motor deficits and early complete recovery of these deficits were noted in $72.6 \%$ (53/73) of these patients.

Majority of the studies on ACDF have graded neurological deficits using various scores like Nurick's grade, JOA, modified JOA scores etc. which combine both sensory and motor deficits. $(4,8,16$, 19) Detailed assessment of motor deficits with grading of motor power for various muscle groups has not been done in most of the studies on ACDF. $(8,16)$ In the present study we compared the preoperative motor power and early postoperative (at the time of discharge) motor power following ACDF in all major groups of muscles of lower and upper extremity and found that significant improvement in motor power in early postoperative period. Long term follow-up studies in patients following ACDF is required as they can develop new deficits secondary to adjacent segment disease. (16)

\section{CONCLUSION}

Early improvement in preoperative motor deficits can be expected in majority of the patients with degenerative cervical PIVD following ACDF.

\section{REFERENCES}

1. Bhaganagare AS, Nagesh SA, Shrihari BG, Naik V, Nagarjun MN, Pai BS: Management of cervical monoradiculopathy due to prolapsed intervertebral disc, an institutional experience. Journal of craniovertebral junction \& spine 8:132, 2017.
2. Boakye M, Mummaneni PV, Garrett M, Rodts G, Haid R: Anterior cervical discectomy and fusion involving a polyetheretherketone spacer and bone morphogenetic protein. Journal of Neurosurgery: Spine 2:521-525, 2005.

3. Bohlman $\mathrm{HH}$, Emery $\mathrm{SE}$, Goodfellow DB, Jones $\mathrm{PK}$ : Robinson anterior cervical discectomy and arthrodesis for cervical radiculopathy. Long-term follow-up of one hundred and twenty-two patients. JBJS 75:1298-1307, 1993.

4. Chiles III BW, Leonard MA, Choudhri HF, Cooper PR: Cervical spondylotic myelopathy: patterns of neurological deficit and recovery after anterior cervical decompression. Neurosurgery 44:762-769, 1999.

5. Chung JY, Park J-B, Seo H-Y, Kim SK: Adjacent Segment Pathology after Anterior Cervical Fusion. Asian Spine J 10:582-592, 2016.

6. Dardis RM, Saxena A, Shad A, Chitnavis B, Gullan R (2012) Disc Replacement Technologies in the Cervical and Lumbar Spine. Elsevier, pp 1777-1788.

7. Davis RA: A long-term outcome study of 170 surgically treated patients with compressive cervical radiculopathy. Surgical neurology 46:523-533, 1996.

8. Emery SE, Bohlman HH, Bolesta MJ, Jones PK: Anterior cervical decompression and arthrodesis for the treatment of cervical spondylotic myelopathy. Two to seventeen-year follow-up. JBJS 80:941-951, 1998.

9. Fountas KN, Kapsalaki EZ, Nikolakakos LG, Smisson HF, Johnston KW, Grigorian AA, Lee GP, Robinson Jr JS: Anterior cervical discectomy and fusion associated complications. Spine 32:2310-2317, 2007.

10. Gore DR, Sepic SB: Anterior Discectomy and Fusion for Painful Cervical Disc Disease: A Report of 50 Patients With an Average Follow-up of 21 Years. Spine 23:2047-2051, 1998.

11. Hessler C, Boysen $K$, Westphal M, Regelsberger J: Functional and radiological outcome after ACDF in 67 cases. Zeitschrift fur Orthopadie und Unfallchirurgie 149:683-687, 2011.

12. Jacobs WC, Anderson PG, Limbeek J, Willems PC, Pavlov $P$ : Single or double-level anterior interbody fusion techniques for cervical degenerative disc disease. Cochrane Database Syst Rev 4:CD, 2004.

13. Jagannathan J, Shaffrey $\mathrm{Cl}$, Oskouian RJ, Dumont AS, Herrold C, Sansur CA, Jane Sr JA: Radiographic and clinical outcomes following single-level anterior cervical discectomy and allograft fusion without plate placement or cervical collar. 2008.

14. Kim W-K (2011) Role of Minimally Invasive Cervical Spine Surgery in the Aging Spine. Elsevier, pp 198-203.

15. Lebl DR, Hughes A, Cammisa FP, O'Leary PF: Cervical spondylotic myelopathy: pathophysiology, clinical presentation, and treatment. HSS journal 7:170-178, 2011.

16. Lehmann $\mathrm{CL}$, Buchowski JM, Stoker GE, Riew KD: Neurologic Recovery after Anterior Cervical Discectomy and Fusion. Global Spine J 4:41-46, 2014.

17. Levinthal R: Anterior cervical discectomy with and 
without fusion. Results, complications, and long-term follow-up. Spine 19:2343-2347, 1994.

18. Murrey D, Janssen M, Delamarter R, Goldstein J, Zigler J, Tay B, Darden B: Results of the prospective, randomized, controlled multicenter Food and Drug Administration investigational device exemption study of the ProDisc-C total disc replacement versus anterior discectomy and fusion for the treatment of 1-level symptomatic cervical disc disease. The Spine Journal 9:275-286, 2009.

19. Nurjck S: The pathogenesis of the spinal cord disorder associated with cervical spondylosis. Brain 95:87-100, 1972.

20. Orr RD, Zdeblick TA: Cervical Spondylotic Myelopathy: Approaches to Surgical Treatment. Clinical orthopaedics and related research 359:58-66, 1999.

21. Saldua NS, Okafor C, Harris EB, Vaccaro AR (2011) The Role of Spinal Fusion and the Aging Spine: Stenosis without Deformity. Elsevier, pp 329-335.

22. Truumees E, Herkowitz HN: Cervical spondylotic myelopathy and radiculopathy. Instr Course Lect 49:339360, 2000.

23. Wu T-k, Wang B-y, Deng M-d, Hong Y, Rong X, Chen H, Meng $Y$, Liu $\mathrm{H}$ : A comparison of anterior cervical discectomy and fusion combined with cervical disc arthroplasty and cervical disc arthroplasty for the treatment of skip-level cervical degenerative disc disease: A retrospective study. Medicine 962017.

24. Yolas C, Ozdemir NG, Okay HO, Kanat A, Senol M, Atci IB, Yilmaz H, Coban MK, Yuksel MO, Kahraman U: Cervical disc hernia operations through posterior laminoforaminotomy. Journal of Craniovertebral Junction and Spine 7:91, 2016.

25. Yu J, Ha Y, Shin J, Oh JK, Lee CK, Kim KN, Yoon DH: Influence of plate fixation on cervical height and alignment after one-or two-level anterior cervical discectomy and fusion. British journal of neurosurgery:18, 2017. 